\title{
Simulation and experiment study on an inductor for DC/DC converter in charging cord
}

\author{
Dian Yuwen ${ }^{1, \mathrm{a}}$, Xu Yang ${ }^{1}$, Mofan $\operatorname{Tian}^{1}$, Linlin $\operatorname{Tan}^{1}$ and Zhenghao Guo ${ }^{2}$ \\ ${ }^{1}$ State Key Laboratory of Electrical Insulation and Power Equipment ,710049 Xi'an Jiao tong University, Xi'an, \\ Shaanxi, China \\ ${ }^{2}$ College of Information Science and Technology, 100875 Beijing Normal University, Beijing, China
}

\begin{abstract}
The purpose of the study is to design an inductor for DC/DC converter in charging cord. In an integrated circuit(IC), the size of capacitor and resistance is easy to reduce. Thus the key is to guarantee the size and value of the inductor simultaneously. To fit the cord, the new inductor is shaped as a cylinder. The COMSOL Multiphysics is used as the simulation tool for the characterization of the inductor and the comparison of the influence of different sizes of copper core on inductance value. It proves that the inductor is practical in DC/DC converter based on the experiment.
\end{abstract}

Keywords: DC/DC converter; inductor; electromagnetic simulation; charging cord.

\section{Introduction}

As the widespread use of electronic devices, such as smart phone and iPad, people are getting more and more dependent on the mobile applications which bring a lot of convenience to everybody. However, numerous mobile applications consume a large part of the power in the cell phone. Meanwhile, as the development of the electronic devices, their size is much larger, and performance is more diverse. These changes encourage the consumption of the battery, so people have to be getting in the troubles of frequent charging to satisfy the requirements of long-time continuous use of the electronic devices. Most of manufacturers can only expand battery capacity for no technical breakthroughs of lithium battery that is widely adopted in cell phone, which calls for longer charging time. The most annoying problem is that the temperature of devices will become higher when charging time increases, which will shorten the life of electronic devices. In addition, the smart phone mainly uses non-dismantle battery that is bad for cooling. In general, power adapters and some electrical devices are in charge of voltage conversion while USB power lines are popular for lowvoltage electrical devices to transmit data and charge. Therefore, converters not only raise the temperature of devices, but also limit the further size reduction of power adapters.

This paper presents that it can put the DC/DC converter in a cord to decrease the calorific power and the size of power adapter and electronic devices. The most difficult of this part is how to design an inductor that meets the demands of the size and value of the inductor simultaneously. Thus, the purpose of the study is to design the inductor for DC/DC converter which is embedded in charging cord based on the simulation and experiment.

\footnotetext{
a Corresponding author: yuwendian@mail.xjtu.edu.cn
} 


\section{Parameter calculation}

\subsection{Derivation of inductor value}

The buck converter power stage model is shown as Figure 1.

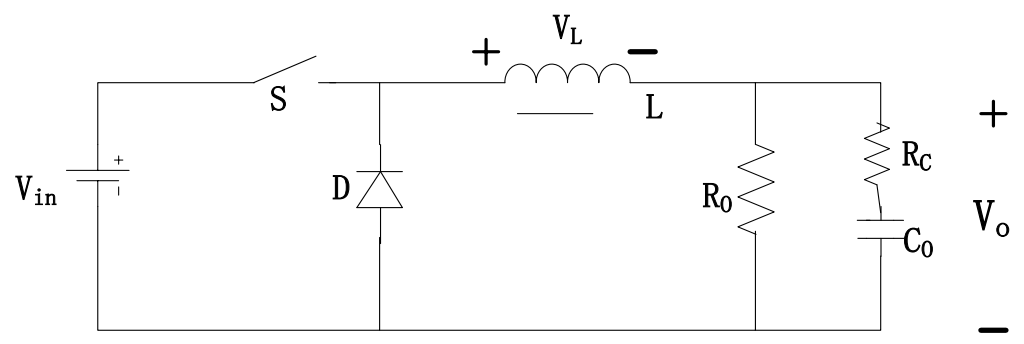

Figure 1. The buck converter power stage model

According to the definition of inductor,

$$
L=N \frac{d \Phi}{d i}
$$

$L$ is the value of inductor; $N$ is the turns of the inductor; $i$ is the current through the inductor; $\Phi$ is the flux in the inductor.

Differentiate both sides of the equation by $d t$, we can get the equation of inductor voltage.

$$
L \frac{d i}{d t}=N \frac{d \Phi}{d t}=v
$$

If we assume that the input voltage $V_{i}$ and output voltage $V_{\text {out }}$ are constant whether the switch is on or off. Then the inductor voltage is gained.

The inductor voltage when the switch turns on is $V_{\text {on }}$.

$$
V_{\text {on }}=V_{i}-V_{s}-V_{o}
$$

The inductor voltage when the switch turns off is $V_{\text {off }}$.

$$
V_{\text {off }}=-V_{D}-V_{o}
$$

In the equation, $V_{s}$ is the turn-on voltage of the switch; $V_{D}$ is the turn-on voltage of the diode. Since $V_{s}$ and $V_{D}$ are so small, we can ignore them when calculating. So

$$
\begin{aligned}
& V_{\text {on }}=V_{i}-V_{o} \\
& V_{\text {off }}=-V_{o}
\end{aligned}
$$

It is clear that $V_{i}$ and $V_{\text {out }}$ are constant, so $L \frac{d i}{d t}=v=$ const . That is 


$$
\frac{d i}{d t}=\frac{v}{L}=\text { const }
$$

Replace $\frac{d i}{d t}$ with $\frac{\Delta i}{\Delta t}$, we can obtain

$$
L=\frac{\Delta t}{\Delta i} v
$$

$\Delta i$ is taken as the ripple current in the inductor. We can derive the value of inductor from the equation:

When the switch turns on

$$
L=\frac{t_{\text {on }}}{\Delta i_{\text {on }}}\left(V_{i}-V_{o}\right)
$$

When the switch turns off

$$
L=-\frac{t_{\text {off }}}{\Delta i_{\text {off }}} V_{i}
$$

$\Delta i_{\text {on }}$ is the turn-on ripple current ; $t_{\text {on }}$ is the turn-on time ; $\Delta i_{\text {off }}$ is the turn-off ripple current ; $t_{\text {off }}$ is the turn-off time.

In a steady power supply,

$$
\Delta i_{\text {on }}=-\Delta i_{\text {off }}=\Delta i_{L}
$$

$\Delta i_{L}$ is the magnitude of ripple current in the inductor. We can derive from equation

$$
\frac{t_{\text {on }}}{t_{\text {off }}}=\frac{V_{o}}{V_{i}-V_{o}}
$$

Then

$$
\begin{gathered}
\frac{t_{\text {on }}}{t_{\text {on }}+t_{\text {off }}}=t_{\text {on }} \cdot f_{s}=\frac{V_{o}}{V_{i}} \\
t_{\text {on }}=\frac{V_{o}}{V_{i} \cdot f_{s}}
\end{gathered}
$$

$f_{s}$ is the switch frequency of the buck circuit. Finally we can obtain the equation of $L$.

$$
L=\frac{V_{o}(1-D)}{f_{s} \cdot \Delta i_{L}}
$$

$D$ is the duty cycle of the buck circuit. 


\subsection{The structure and geometric parameter of inductor}

In order to fit the shape of the charging cord, the inductor is designed as a cylinder. The cross-section of the inductor is shown in Figure 2.
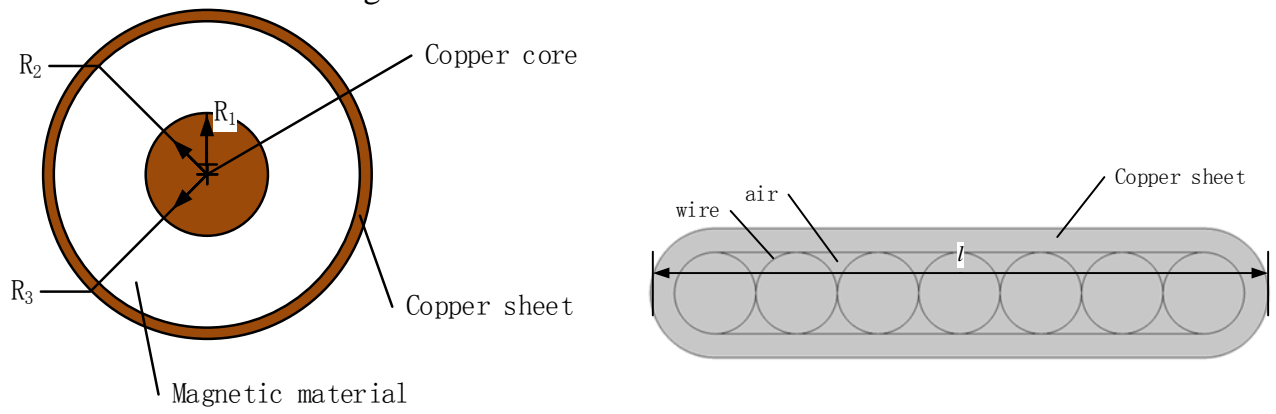

Figure 2. The cross section(left) and the vertical section(right) of the inductor

In Figure 2, $\mathrm{R}_{1}$ is the radius of copper core; $\mathrm{R}_{2}$ is the radius of magnetic material; $\mathrm{R}_{3}$ is the radius of the copper sheet; $l$ is the length of the inductor. We can determine these geometric parameters by the equation (16).

$$
\begin{aligned}
L & =L_{i}+L_{o}+L_{i}^{\prime} \\
& =\frac{\mu_{0} \mu_{r} l}{8 \pi}+\frac{\mu_{0} \mu_{r} l}{2 \pi} \ln \left(\frac{R_{2}}{R_{1}}\right)+\frac{\mu_{0} \mu_{r} l}{2 \pi}\left[\left(\frac{R_{3}^{2}}{R_{3}^{2}-R_{2}^{2}}\right)^{2} \ln \left(\frac{R_{3}}{R_{1}}\right)-\frac{R_{3}^{2}}{R_{3}^{2}-R_{2}^{2}}+\frac{1}{4} \frac{R_{3}^{2}+R_{2}^{2}}{R_{3}^{2}-R_{2}^{2}}\right]
\end{aligned}
$$

$L$ is the value of inductor; $L_{i}$ is the internal magnetic inductance of copper core; Lo is the external magnetic inductance of copper core; $L_{i}{ }^{\prime}$ is the internal magnetic inductance of copper sheet; $\mu_{0}$ is the permeability of vacuum; $\mu_{r}$ is the relative permeability of magnetic material.

\section{Simulation of inductance using COMSOL}

COMSOL Multiphysics is model based software, which solves both stationary and time-dependent partial differential equations (PDEs), by numerical techniques based on the finite element method. The COMSOL Multiphysics simulation software environment facilitates all steps in the modelling process-defining model geometry, meshing, specifying required physics, solving, and then visualizing results.

In this paper, we choose a buck converter whose power is $24 \mathrm{~W}$, switch frequency is $1 \mathrm{MHz}$, input voltage is $12 \mathrm{~V}$, output voltage ranges from $1 \mathrm{~V}$ to $5 \mathrm{~V}$. The ripple factor is 0.2 . Therefore, we can calculate the value of inductor from equation (15). The inductance needed is $7.5 \mu \mathrm{H}$. The model uses the 2D analysis of magnetic field of the AC/DC module in COMSOL Multiphysics 5.0 software tool. The model is the cross section of the inductor and consists of three-layer annulus which is shown in Figure 2. The second layer is filled with a kind of magnetic material whose relative permeability is 45 . The first and the third layers are filled with copper. The radius and the thickness of the copper sheet are $4.5 \mathrm{~mm}$ and $0.1 \mathrm{~mm}$. The inductance changes with the radius of the copper core. Compare the theoretical value with the simulative value of the inductor, then we can obtain the final geometric parameters of the inductor.

The model was meshed using the default mesh parameter and the mesh consists of 6254 elements as shown in Figure 3. 


\section{AEST2016}

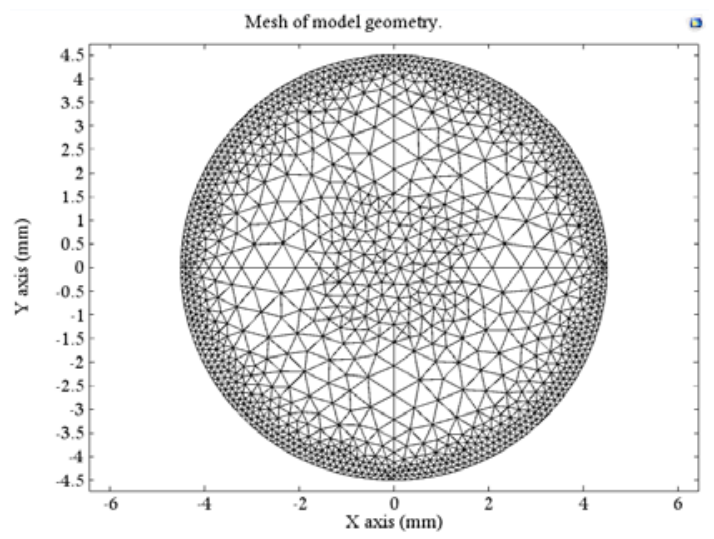

Figure 3. The mesh of the inductor's cross section

The distribution diagram of magnetic flux density is shown in Figure 4. It is evident from the figure that the magnetic flux density has a descended distribution trend from the centre to the periphery of the magnetic material.

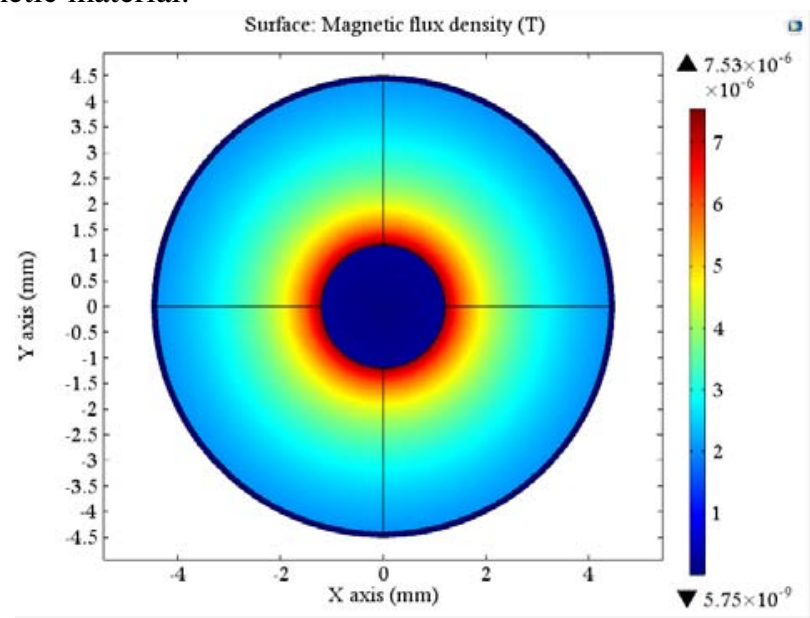

Figure 4. The magnetic flux density of the inductor's cross section

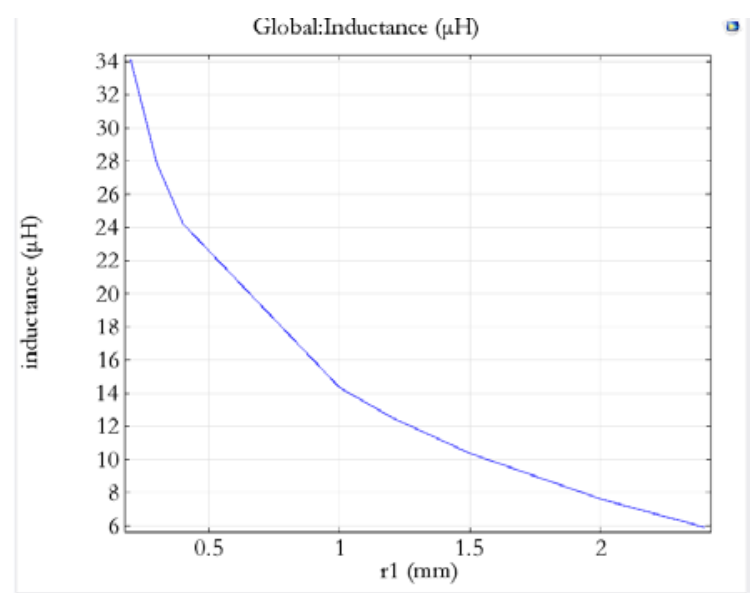

Figure 5. The inductance changes with the radius of the copper core r1 
It shows that the inductance changes with the radius of the copper core in Figture 5. According to the result of the simulation, if the length of the inductor is $1 \mathrm{~mm}$, the inductance is $7.6181 \mu \mathrm{H}$ when the radius of copper core is $2 \mathrm{~mm}$. The comparison of theoretical and simulative value is shown in Table 1. The final geometric parameters of inductor are listed in Table 2.

Table 1. The comparison of inductance

\begin{tabular}{|c|c|}
\hline Different values & $\mu \mathrm{H}$ \\
\hline theoretical value & 0.5000 \\
\hline simulative value & 0.5181 \\
\hline
\end{tabular}

Table 2. The final geometric parameters of inductor

\begin{tabular}{|c|c|c|c|c|}
\hline geometric parameters & $l$ & $\mathrm{R}_{1}$ & $\mathrm{R}_{2}$ & $\mathrm{R}_{3}$ \\
\hline$(\mathrm{mm})$ & 1 & 2 & 4.4 & 4.5 \\
\hline
\end{tabular}

\section{Experiment results}

The experiment has been done to verify the practicability of the above inductor based on MPQ8633. The corresponding parameters are as following : $V_{i n}=12 \mathrm{~V}, V_{O}=1 \mathrm{~V}, f_{s}=1 \mathrm{MHz}, \mathrm{L}=0.5 \mu \mathrm{H}$, no load.

Figure 6 shows the experiment waveforms of output voltage $V o$ and inductor current $I_{L}$. The maxium calculated ripple current is 2A. It can be seen that the ripple current of $I_{L}$ is smller the maxium ripple current. The practicability of the buck circuit with the new inductor is verified.

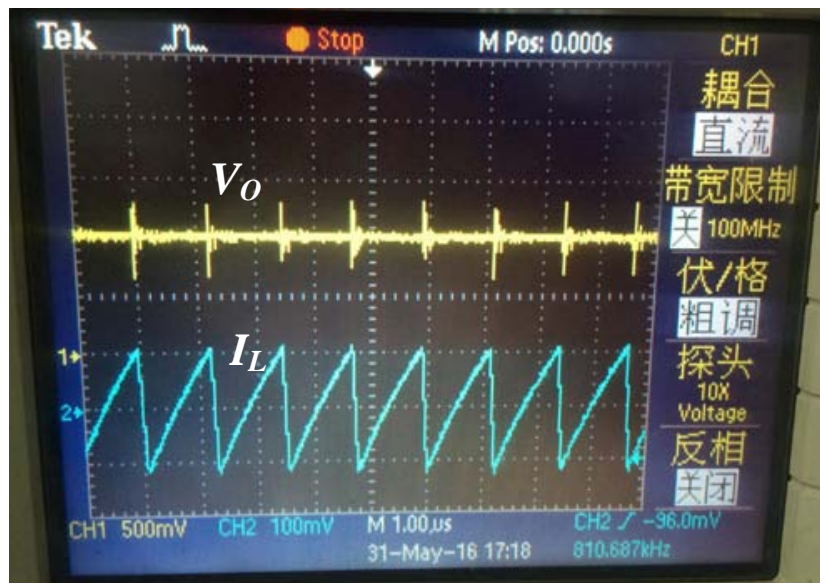

Figure 6. Experiment waveforms of $V_{O}$ and $I_{L}$

\section{Conclusion}

Simulation and experiment research has been made on a buck circuit with a cylindrical inductor in this paper. The design principle of the inductor in a buck converter is analysed in detail. For the practicability, the inductor is designed as axial symmetry. So we can calculate the geometric parameters by the simulation of the cross section of the inductor using COMSOL Multiphysics. The 
inductor is satisfied with the requirements of size and inductance. According to the results of the experiment, it is verified that we can use this new tiny inductor in the DC/DC converter.

\section{References}

1. R. Pradhan, A. Mitra, S. Das, IEEE Intl. Conf. Sys. Med. Bio., 37 (2010).

2. L. I. ZhengHui, X. I. Zi-Qiang, J. Hubei Univ. Tech. (2007).

3. Y. Liu, G. Yao, X. He, H. Wu, IECON '03, 1981 (2003).

4. S. Kumar, Y. K. Jain, IEEE Sensors J. 15, 1 (2015).

5. Y. Li, P. Ge, B. Niu, Res. J. App. Sci. Eng. \& Tech. 5, 4510 (2013).

6. M. P. Kumar, P. Ponnambalam, S. Sreejith, J. Belwinedward, IEEE Pedes, (2014).

7. Shan T, Wang X, Sheng T, IEEE Int. Conf. Mech. \& Auto., (2015).

8. Y. Li, P. Ge, B. Niu, Res. J. App. Sci. Eng. \& Tech. 5, 4510 (2013).

9. McLyman, CWT. Transformer and inductor design handbook (2016). 